\title{
Off-resonant entanglement generation in a lossy cavity
}

\author{
F. Francica, ${ }^{1}$ S. Maniscalco,${ }^{2}$ J. Piilo, ${ }^{2}$ F. Plastina, ${ }^{1}$ and K.-A. Suominen ${ }^{2}$ \\ ${ }^{1}$ Dip. Fisica, Università della Calabria, 8 INFN - Gruppo collegato di Cosenza, 87036 Arcavacata di Rende (CS) Italy \\ ${ }^{2}$ Department of Physics and Astronomy, University of Turku, FI-20014 Turku, Finland
}

(Dated: November 9, 2018)

\begin{abstract}
We provide an exact and complete characterization of the entanglement dynamics of two qubits coupled to a common structured reservoir at zero temperature. We derive the conditions to maximize reservoir-induced entanglement for an initially factorized state of the two-qubit system. In particular, when the two qubits are placed inside a lossy cavity, we show that high values of entanglement can be obtained, even in the bad cavity limit, in the dispersive regime. Finally we show that, under certain conditions, the entanglement dynamics exhibits quantum beats and we explain their physical origin in terms of the interference between two different transitions coupling the dressed states of the system.

PACS numbers: 03.67.Bg, 03.65.Yz
\end{abstract}

\section{INTRODUCTION}

Quantum entanglement is the powerful resource lying at the root of a new class of technologies based on the laws of quantum theory. The coherent manipulation of quantum systems involves very delicate procedures since the inevitable interaction with their surroundings leads to a loss of information that causes both the transformation of quantum superpositions into statistical mixtures, a process called decoherence, and the disappearance of quantum entanglement in composite systems.

Recently, it has been shown that entanglement can be lost completely in a finite time despite the fact that complete decoherence only occurs asymptotically. This phenomenon, named entanglement sudden death, has been theoretically predicted by $\mathrm{Yu}$ and Eberly [1], and experimentally observed for entangled photon pairs 2] and atomic ensembles [3]. Typically, entanglement sudden death occurs when the two qubits interact with two independent environments as for the case, e.g., of two entangled qubits placed inside two different cavities. For such a configuration, a class of states has been identified which do not experience a complete entanglement loss despite the interaction with local vacuum environments [4]. However, for finite temperature environments the sudden death occurs almost independently of the initial state of the qubit pair [5], although with details that can depend on the amount of non-Markovianity of the environments [6]. In this context, a deeper understanding of the sudden death process has been gained by looking at the quantum correlations shared by the environments which show a sudden birth (though with a quite counterintuitive timing) [7, 8].

A completely different phenomenology emerges when the qubits interact with the same environment. In this case, indeed, entanglement can be created starting from a factorized state or it can even revive after a sudden death. This is due to the effective qubitqubit interaction mediated by the common reservoir [9, 10, 11, 12, 13, 14, 15, 16]. Many theoretical papers have studied reservoir-induced entanglement in the
Markovian regime, that is when the coupling between the qubits and the environment is weak enough to neglect the feedback of information from the reservoir into the system (memoryless dynamics). An interesting extension to these approaches, that goes beyond the Born-Markov approximation, has been presented in Ref. [17].

In a recent paper, we have studied the dynamics of two qubits coupled to a common structured environment using an exact approach that does not rely on the Born-Markov approximation [18]. We focused on the case in which the qubits were identical and resonant with the cavity field, whose spectrum was modelled as a Lorentzian. In this paper we extend our analytical approach to describe the more general situation in which the qubit frequencies are different and non-resonant with the main mode supported by the cavity. Our new analytical results allow us to characterize completely and exactly the entanglement dynamics for a generic initial two-qubit state containing one excitation. We study the time evolution of the entanglement and its dependence on several parameters, all in principle adjustable in the experiments: the relative coupling between the atoms and the cavity field, the initial amount of entanglement, the frequency of the qubits, the detuning from the cavity field, and the quality factor of the cavity. In this way we determine the conditions to achieve maximal reservoirinduced entanglement generation for an initial factorized state of the qubits, and to minimize the loss of entanglement for an initial entangled state.

Depending on the matching of the qubit frequencies, we will distinguish two scenarios displaying different qualitative long time behavior. If the two qubits have the same transition frequency $\left(\omega_{1}=\omega_{2}\right)$, a decoherencefree state (subradiant state) exists [19, 20]. Due to the presence of such a dark state, a non-zero asymptotic entanglement can be obtained in this case. On the other hand, if the two qubits have different transition frequencies $\left(\omega_{1} \neq \omega_{2}\right)$, no subradiant state exists, so that the stationary entanglement always vanishes. For the sake of brevity, we refer to these two cases as subradiant and non-subradiant scenario, respectively. 
One of our main results is the demonstration that high values of reservoir-induced entanglement can be obtained in the dispersive regime even in the bad cavity limit. In general, in this regime the dynamics of the concurrence (that we employ to quantify entanglement [21]) is characterized by a quasi-regular and quasi-periodical pattern since the cavity photon is only virtually excited and therefore the two-qubit system is less affected by the cavity losses. Finally, in the good cavity limit, we predict the occurrence of quantum beats of entanglement and explain their physical origin.

The paper is structured as follows. In Sec. II we present the microscopic Hamiltonian model, for which the exact analytical solution is presented in Sec. III, where we focus on the case in which the spectrum of the environment is Lorentzian as, e.g., for the electromagnetic field inside a lossy resonator. In Sec. IV and V we present and discuss our main results by looking at the entanglement dynamics in the subradiant and nonsubradiant scenarios, respectively, for different coupling regimes and different initial states. Finally, Sec. VI contains summary and conclusions.

\section{THE MODEL}

We study an open quantum system consisting of two qubits coupled to a common zero-temperature bosonic reservoir in the vacuum. The Hamiltonian describing the total system is given by

$$
H=H_{S}+H_{R}+H_{\text {int }},
$$

where $H_{S}$ is the Hamiltonian of the qubits system coupled, via the interaction Hamiltonian $H_{\text {int }}$, to the common reservoir, whose Hamiltonian is $H_{R}$.

The Hamiltonian for the total system, in the dipole and the rotating-wave approximations, can be written as (assuming $\hbar=1$ )

$$
\begin{aligned}
H_{S} & =\omega_{1} \sigma_{+}^{(1)} \sigma_{-}^{(1)}+\omega_{2} \sigma_{+}^{(2)} \sigma_{-}^{(2)} \\
H_{R} & =\sum_{k} \omega_{k} b_{k}^{\dagger} b_{k} \\
H_{\mathrm{int}} & =\left(\alpha_{1} \sigma_{+}^{(1)}+\alpha_{2} \sigma_{+}^{(2)}\right) \sum_{k} g_{k} b_{k}+\text { h.c. }
\end{aligned}
$$

where $b_{k}^{\dagger}, b_{k}$ are the creation and annihilation operators of quanta of the reservoir, $\sigma_{ \pm}^{(j)}$ and $\omega_{j}$ are the inversion operators and transition frequency of the $j$-th qubit $(j=$ $1,2)$; finally $\omega_{k}$ and $\alpha_{j} g_{k}$ are the frequency of the mode $k$ of the reservoir and its coupling strength with the $j$-th qubit.

Here, the $\alpha$ 's are dimensionless real coupling constants measuring the interaction strength of each single qubit with the reservoir. In particular, we assume that these two constants can be varied independently. In the case of two atoms inside a cavity, e.g., this can be achieved by changing the relative position of the atoms in the cavity field standing wave. We denote with $\alpha_{T}=\left(\alpha_{1}^{2}+\alpha_{2}^{2}\right)^{1 / 2}$ the collective coupling constant and with $r_{j}=\alpha_{j} / \alpha_{T}$ the relative interaction strength.

\section{A. Dynamics of the qubit system}

We assume that initially the qubit system and the reservoir are disentangled. We restrict ourselves to the case in which only one excitation is present in the system and the reservoir is in the vacuum. In this case the initial state for the whole system can be written as

$$
|\Psi(0)\rangle=\left[c_{01}|1\rangle_{1}|0\rangle_{2}+c_{02}|0\rangle_{1}|1\rangle_{2}\right] \bigotimes_{k}\left|0_{k}\right\rangle_{R}
$$

where $|0\rangle_{j}$ and $|1\rangle_{j}(j=1,2)$ are the ground and excited states of the $j$-th qubit, respectively, while $\left|0_{k}\right\rangle_{R}$ is the state of the reservoir with zero excitations in the mode $k$.

The time evolution of the total system, under the action of this Hamiltonian, is given by

$$
\begin{aligned}
|\Psi(t)\rangle= & c_{1}(t)|1\rangle_{1}|0\rangle_{2}|0\rangle_{R}+c_{2}(t)|0\rangle_{1}|1\rangle_{2}|0\rangle_{R}+ \\
& +\sum_{k} c_{k}(t)|0\rangle_{1}|0\rangle_{2}\left|1_{k}\right\rangle_{R},
\end{aligned}
$$

where $\left|1_{k}\right\rangle_{R}$ is the state of the reservoir with only one excitation in the $k$-th mode and $|0\rangle_{R}=\bigotimes_{k}\left|0_{k}\right\rangle$.

The reduced density matrix describing the two-qubit systems, obtained from the density operator $|\Psi(t)\rangle\langle\Psi(t)|$ after tracing over the reservoir degrees of freedom, takes the form

$$
\rho(t)=\left(\begin{array}{cccc}
0 & 0 & 0 & 0 \\
0 & \left|c_{1}(t)\right|^{2} & c_{1}(t) c_{2}^{*}(t) & 0 \\
0 & c_{1}^{*}(t) c_{2}(t) & \left|c_{2}(t)\right|^{2} & 0 \\
0 & 0 & 0 & 1-\left|c_{1}\right|^{2}-\left|c_{2}\right|^{2}
\end{array}\right)
$$

The two-qubit dynamics is therefore completely characterized by the amplitudes $c_{1,2}(t)$.

Introducing the $j$-qubit detuning from the mode $k$, $\delta_{k}^{(j)}=\omega_{j}-\omega_{k}$, the equations for the probability amplitudes take the form

$$
\begin{aligned}
& \dot{c}_{j}(t)=-i \alpha_{j} \sum_{k} g_{k} e^{i \delta_{k}^{(j)} t} c_{k}(t), \quad j=1,2 \\
& \dot{c}_{k}(t)=-i g_{k}^{*}\left[\alpha_{1} e^{-i \delta_{k}^{(1)} t} c_{1}(t)+\alpha_{2} e^{-i \delta_{k}^{(2)} t} c_{2}(t)\right] .
\end{aligned}
$$

Formally integrating Eq. (9) and inserting its solution into Eqs. (8), one obtains two integro-differential equations for $c_{1,2}(t)$,

$$
\begin{aligned}
\dot{c}_{1}(t)= & -\sum_{k} \int_{0}^{t} d t_{1}\left[\alpha_{1}^{2}\left|g_{k}\right|^{2} e^{i \delta_{k}^{(1)}\left(t-t_{1}\right)} c_{1}\left(t_{1}\right)\right. \\
& \left.+\alpha_{1} \alpha_{2}\left|g_{k}\right|^{2} e^{i \delta_{k}^{(1)} t} e^{-i \delta_{k}^{(2)} t_{1}} c_{2}\left(t_{1}\right)\right]
\end{aligned}
$$




$$
\begin{aligned}
\dot{c}_{2}(t)= & -\sum_{k} \int_{0}^{t} d t_{1}\left[\alpha_{1} \alpha_{2}\left|g_{k}\right|^{2} e^{i \delta_{k}^{(2)} t} e^{-i \delta_{k}^{(1)} t_{1}} c_{1}\left(t_{1}\right)\right. \\
& \left.+\alpha_{2}^{2}\left|g_{k}\right|^{2} e^{i \delta_{k}^{(2)}\left(t-t_{1}\right)} c_{2}\left(t_{1}\right)\right] .
\end{aligned}
$$

In the continuum limit for the reservoir spectrum the sum over the modes is replaced by the integral

$$
\sum_{k}\left|g_{k}\right|^{2} \rightarrow \int d \omega J(\omega)
$$

where $J(\omega)$ is the reservoir spectral density. In the following we focus on the case in which the structured reservoir is the electromagnetic field inside a lossy cavity. In this case, the fundamental mode supported by the cavity displays a Lorentzian broadening due to the non-perfect reflectivity of the cavity mirrors. Hence the spectrum of the field inside the cavity can be modelled as

$$
J(\omega)=\frac{W^{2}}{\pi} \frac{\lambda}{\left(\omega-\omega_{c}\right)^{2}+\lambda^{2}},
$$

where the weight $W$ is proportional to the vacuum Rabi frequency and $\lambda$ is the width of the distribution and therefore describes the cavity losses (photon escape rate).

We now introduce the correlation function $f\left(t-t_{1}\right)$, defined as the Fourier transform of the reservoir spectral density $J(\omega)$,

$$
f\left(t-t_{1}\right)=\int d \omega J(\omega) e^{i\left(\omega_{c}-\omega\right)\left(t-t_{1}\right)},
$$

where $\omega_{c}$ is the fundamental frequency of the cavity. In terms of the correlation function Eqs. (10)-(11) become

$$
\begin{aligned}
\dot{c}_{1}(t)= & -\int_{0}^{t} d t_{1}\left[\alpha_{1}^{2} c_{1}\left(t_{1}\right)+\alpha_{1} \alpha_{2} c_{2}\left(t_{1}\right) e^{-i \delta_{21} t_{1}}\right] \\
& \times f\left(t-t_{1}\right) e^{i \delta_{1}\left(t-t_{1}\right)} \\
\dot{c}_{2}(t)= & -\int_{0}^{t} d t_{1}\left[\alpha_{1} \alpha_{2} c_{1}\left(t_{1}\right) e^{i \delta_{21} t_{1}}+\alpha_{2}^{2} c_{2}\left(t_{1}\right)\right] \\
& \times f\left(t-t_{1}\right) e^{i \delta_{2}\left(t-t_{1}\right)},
\end{aligned}
$$

where $\delta_{j}=\omega_{j}-\omega_{c}$ and $\delta_{21}=\omega_{2}-\omega_{1}$.

Performing the Laplace transform of Eqs. (13)-(14) yields

$$
\begin{aligned}
s \widetilde{c}_{1}(s)-c_{1}(0)= & -\left[\alpha_{1}^{2} \widetilde{c}_{1}(s)+\alpha_{1} \alpha_{2} \widetilde{c}_{2}\left(s+i \delta_{21}\right)\right] \\
& \times \widetilde{f}\left(s-i \delta_{1}\right), \\
s \widetilde{c}_{2}(s)-c_{2}(0)= & -\left[\alpha_{1} \alpha_{2} \widetilde{c}_{1}\left(s-i \delta_{21}\right)+\alpha_{2}^{2} \widetilde{c}_{2}(s)\right] \\
& \times \widetilde{f}\left(s-i \delta_{2}\right) .
\end{aligned}
$$

From the equations above one can derive the quantities $\widetilde{c}_{1}(s)$ and $\widetilde{c}_{2}(s)$. Finally, inverting the Laplace transform one obtains a formal solution for the amplitudes $c_{1}(t)$ and $c_{2}(t)$. The main steps for deriving the general solution are outlined in Appendix A. For specific forms of the reservoir spectral density, as the one we consider in this paper, it is possible to obtain simple analytic expressions for these coefficients.

Before discussing the general features of the dynamics we notice that, when the two qubits have the same transition frequency, $\omega_{1}=\omega_{2}$, a subradiant, decoherence-free state exists, that does not decay in time. The existence of the subradiant state does not depend on the form of the spectral density and therefore on the resonance/offresonance condition. Such a state takes the form

$$
\left|\psi_{-}\right\rangle=r_{2}|1\rangle_{1}|0\rangle_{2}-r_{1}|0\rangle_{1}|1\rangle_{2} .
$$

When the two qubits have different frequencies, $\omega_{1} \neq \omega_{2}$, there is no decoherence-free state.

This simple consideration enables us to draw general conclusions about the dynamics of entanglement for long times. Indeed, one can observe two qualitatively different behaviors. In the subradiant scenario, occurring for $\omega_{1}=$ $\omega_{2}$, a subradiant state exists and therefore that part of the initial entanglement stored in $\left|\psi_{-}\right\rangle$will be 'trapped' for arbitrary long times. In the non-subradiant scenario, when $\omega_{1} \neq \omega_{2}$, the subradiant state does not exist. Hence all initial entanglement will decay and is eventually lost for long times.

We now derive the solution for the coefficients $c_{1}(t)$ and $c_{2}(t)$ and study the entanglement dynamics discussing separately the two cases outlined above.

\section{B. Subradiant Scenario}

For $\omega_{1}=\omega_{2}$ the analytical solution for the amplitudes $c_{1}(t)$ and $c_{2}(t)$ takes a simple form, with a structure analogous to the solution of the resonant case presented in Ref. [18],

$$
\begin{aligned}
& c_{1}(t)=\left[r_{2}^{2}+r_{1}^{2} \mathcal{E}(t)\right] c_{1}(0)-r_{1} r_{2}[1-\mathcal{E}(t)] c_{2}(0), \\
& c_{2}(t)=-r_{1} r_{2}[1-\mathcal{E}(t)] c_{1}(0)+\left[r_{1}^{2}+r_{2}^{2} \mathcal{E}(t)\right] c_{2}(0),
\end{aligned}
$$

with

$\mathcal{E}(t)=e^{-(\lambda-i \delta) t / 2}\left[\cosh (\Omega t / 2)+\frac{\lambda-i \delta}{\Omega} \sinh (\Omega t / 2)\right]$,

where $\delta_{1}=\delta_{2} \equiv \delta$ and $\Omega=\sqrt{\lambda^{2}-\Omega_{R}^{2}-i 2 \delta \lambda}$, with $\Omega_{R}=\sqrt{4 W^{2} \alpha_{T}^{2}+\delta^{2}}$ the generalized Rabi frequency and $\mathcal{R}=W \alpha_{T}$ the vacuum Rabi frequency.

As in the resonant case, the state $\left|\psi_{-}\right\rangle$does not evolve in time and the only relevant time evolution is the one of its orthogonal superradiant state

$$
\left|\psi_{+}\right\rangle=r_{1}|1\rangle_{1}|0\rangle_{2}+r_{2}|0\rangle_{1}|1\rangle_{2} .
$$


The function $\mathcal{E}(t)$ is the survival amplitude of the superradiant state $\left\langle\psi_{+}(t) \mid \psi_{+}(0)\right\rangle=\mathcal{E}(t)$. If we express the initial state of the qubits as a superposition of $\left|\psi_{ \pm}\right\rangle$, that is $|\psi(0)\rangle=\beta_{-}\left|\psi_{-}\right\rangle+\beta_{+}\left|\psi_{+}\right\rangle$with $\beta_{ \pm}=\left\langle\psi_{ \pm} \mid \psi(0)\right\rangle$, we see that, while part of the initial state will be trapped in the subradiant state $\left|\psi_{-}\right\rangle$, another part will decay following Eq. (20). Thus the amount of entanglement that survives depends on the specific initial state and on the value of the coefficients $r_{j}$.

\section{Non-subradiant Scenario}

For $\omega_{1} \neq \omega_{2}$ no subradiant or decoherence-free state exists and, as a consequence, the analytical expression for the amplitudes $c_{1,2}(t)$ becomes more complicated

$$
\begin{aligned}
& c_{1}(t)=\mathcal{E}_{11}\left(t ; r_{1}\right) c_{1}(0)+\mathcal{E}_{12}\left(t ; r_{1}\right) c_{2}(0), \\
& c_{2}(t)=\mathcal{E}_{21}\left(t ; r_{1}\right) c_{1}(0)+\mathcal{E}_{22}\left(t ; r_{1}\right) c_{2}(0),
\end{aligned}
$$

where the functions $\mathcal{E}_{i j}\left(t ; r_{1}\right)$ depend not only on time but also on the value of $r_{1}$.

We emphasize that in both scenarios, the solution of the differential equations for the amplitudes $c_{1,2}(t)$ is exact as we have not performed neither the Born nor the Markov approximation. The structure of the functions $\mathcal{E}_{i j}\left(t ; r_{1}\right)$ and the main steps to the solution are briefly outlined in Appendix A.

\section{Dispersive regime}

In this subsection we focus on the system dynamics when the qubits are far off-resonant from the main cavity mode, i.e. for $\delta_{1}, \delta_{2} \gg \mathcal{R}$. In this regime, both in the subradiant and in the non-subradiant scenarios, the main features of the dynamics can be obtained by looking at the effective dispersive Hamiltonian describing the coupling of the two qubits with a single-mode cavity field [22, 23, 24] and remembering that this behavior must then be corrected taking into account the effect of the cavity losses. In Appendix B we derive the effective dispersive Hamiltonian for this system, assuming that the cavity field is initially in the vacuum state,

$H_{e f f}=\sum_{j=1}^{2} \frac{\mathcal{R}^{2} r_{j}^{2}}{\delta_{j}} \sigma_{+}^{(j)} \sigma_{-}^{(j)}+\frac{\mathcal{R}^{2} r_{1} r_{2}}{2 \delta_{j}}\left(\sigma_{+}^{(1)} \sigma_{-}^{(2)}+\sigma_{+}^{(2)} \sigma_{-}^{(1)}\right)$.

The first two terms in the Hamiltonian are proportional to $\sigma_{+}^{(j)} \sigma_{-}^{(j)}$ and describe the Stark shifts due to the dispersive interaction with the cavity vacuum. The remaining terms describe an effective dipole-dipole coupling between the two atoms induced by the cavity mode. As we will see in the following these two terms play an essential role in the entanglement generation process. By looking at Eq. (24) we notice that both the Stark shifts and the effective interaction strength between the qubits are now $\propto \mathcal{R}^{2} / \delta_{1,2}$.
In the dispersive regime the cavity is only virtually excited, thus the photon loss is less important and the effective decoherence rate due to the cavity decay is strongly suppressed to the advantage of the generation of entanglement. As we will see in Sec. III A for the subradiant scenario, the effective decoherence rate due to the cavity decay in this case becomes $\left(\mathcal{R}^{2} / \delta^{2}\right) \lambda$.

\section{ENTANGLEMENT DYNAMICS}

To study the time evolution of the two-qubit entanglement we use the concurrence $C(t)$ [21]. This is an entanglement measure related to the entanglement of formation, ranging from one for maximally entangled states to zero for separable ones.

For the system of two qubits described by the reduced density matrix of Eq. (7) the concurrence takes a very simple form

$$
C(t)=2\left|c_{1}(t) c_{2}^{*}(t)\right|
$$

Such equation shows a relation between the behavior of the concurrence and the time evolution of the excitation shared by the two qubits. Having in mind the considerations of Sec. I D one may understand how, through a suitable choice of the detuning between the qubits and the cavity, it is possible to improve both the generation of entanglement and its preservation for long times.

To better discuss the time evolution of the concurrence as a function of the initial amount of entanglement stored in the system, we consider a general initial states of the form given by Eq. (5) with

$c_{01}=\sqrt{\frac{1-s}{2}}, \quad c_{02}=\sqrt{\frac{1+s}{2}} e^{i \phi}$, with $\quad-1 \leq s \leq 1$.

Here, the separability parameter $s$ is related to the initial concurrence as $s^{2}=1-C(0)^{2}$.

Before describing in detail the dynamics in the subradiant scenario (Sec. III) and non-subradiant scenario (Sec. IV), it is useful to recall the main features of the time evolution of the entanglement when $\omega_{1}=\omega_{2}=\omega_{c}$, i.e. in the resonant case, as discussed in Ref. [18].

(i) The concurrence dynamics, as well as the value of the stationary concurrence, depends on the relative coupling strength, i.e. on the parameter $r_{1}$.

(ii) For certain entangled initial states, there exist at least one time instant $\bar{t}<\infty$ at which $C(\bar{t})=0$, both in the strong coupling (good cavity) and weak coupling (bad cavity) limits, i.e. for $\lambda \ll \mathcal{R}$ and $\lambda \gg \mathcal{R}$, respectively [See Fig. 1 (a)].

(iii) In the weak coupling (bad cavity) limit, for an initially factorized state, the reservoir creates entanglement and this is indicated by a monotonic increase in the value of the concurrence.

(iv) In the weak coupling (bad cavity) limit, for an initially entangled state, the reservoir causes entanglement 
loss and the concurrence decreases with time until reaching, in some cases, the value zero, after which a small fraction of entanglement can be recreated [See Fig. 1] (a)].

(v) In the strong coupling (good cavity) limit, oscillations in the concurrence appear. For an initially factorized state there exist times at which the value of the concurrence is higher than the value of the stationary concurrence.

In the next two sections we are going to study how the time evolution of the concurrence is modified in presence of detuning.

\section{OFF-RESONANT ENTANGLEMENT IN THE SUBRADIANT SCENARIO}

We begin considering the case $\omega_{1}=\omega_{2}$. Whenever possible, rather than discussing the exact expression of the concurrence, we will try to derive simpler approximated expressions which are useful for understanding the physical processes taking place in the system.

\section{A. Bad cavity limit - Enhancement of the entanglement generation}

In the bad cavity case, e.g., for $R=\mathcal{R} / \lambda=0.1$, and for small values of the detuning $\delta<\mathcal{R}$, the behavior of the concurrence does not change appreciably compared to the resonant case. For values of the detuning $\delta \approx \mathcal{R}$, i.e. when approaching the dispersive regime, the dynamics for an initially factorized state $(s=1)$ shows a monotonic increase towards the stationary value of the concurrence as in the resonant case. However, a significant change occurs in the bad cavity limit when the system is prepared in an initial entangled state. Indeed one can prove that in this regime, contrary to the resonant case,

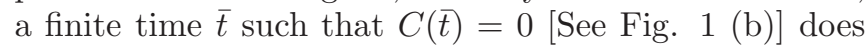
not exist anymore.

We now focus on the dispersive regime $\delta \gg \lambda \gg \mathcal{R}$. If the qubit-system is initially entangled, e.g., for $s=0$, the expression for the concurrence can be simplified as follows

$$
\begin{aligned}
C(t)=|\mathcal{E}| & \approx e^{-\frac{\mathcal{R}^{2}}{\delta^{2}} \lambda t}, \quad \text { for } \quad r_{1}=0,1 ; \\
C(t)=|\mathcal{E}|^{2} & \approx e^{-2 \frac{\mathcal{R}^{2}}{\delta^{2}} \lambda t}, \quad \text { for } \quad r_{1}=1 / \sqrt{2} .
\end{aligned}
$$

The equations above show that the concurrence vanishes with the decay rate $\left(\mathcal{R}^{2} / \delta^{2}\right) \lambda$ when only one of the two qubits is coupled to the environment $\left(r_{1}=0,1\right)$, and with $2\left(\mathcal{R}^{2} / \delta^{2}\right) \lambda$ when both qubits are identically coupled to the environment (for $r_{1}=1 / \sqrt{2}$ ). Since $\mathcal{R} / \delta \ll 1$ this proves that in the dispersive regime the decay of entanglement is strongly inhibited compared to the resonant regime since in this case the two atoms exchange energy only via the virtual excitation of the cavity field and therefore the cavity losses do not affect strongly the dynamics.

For large enough detunings the entanglement shows oscillations as a function of time for all of the initial atomic states for which a finite stationary concurrence is obtained, $C_{s} \neq 0$. Due to the presence of these oscillations and for an initially factorized state, the concurrence reaches values greater than the stationary value $C_{s}$ even in the bad cavity limit, as shown in Fig. 2 For example, for $r_{1}=\sqrt{3} / 2, R=0.1$ and $\delta=10 \lambda$, at $\lambda t \approx 2 \times 10^{3}$ the concurrence reaches the value $C=0.92$. For an initially factorized state $(s=1)$ and for $r_{1}=1 / \sqrt{2}$ we can derive the following approximated expression for the concurrence

$$
C(t) \approx \frac{1}{2} \sqrt{1+e^{-4 \frac{\mathcal{R}^{2}}{\delta^{2}} \lambda t}-2 e^{-2 \frac{\mathcal{R}^{2}}{\delta^{2}} \lambda t} \cos \left(2 \frac{\mathcal{R}^{2}}{\delta} t\right)} .
$$

From this equation one sees that $C(t)$ attains its maximum value at $t=\frac{\pi \delta}{2 \mathcal{R}^{2}}$. This formula also shows that the concurrence undergoes a series of damped oscillations with frequency $2 \mathcal{R}^{2} / \delta$ and decay rate $2(\mathcal{R} / \delta)^{2} \lambda$.

With increasing detuning, the oscillations become more and more regular, quasi-periodic. The pattern is similar to the oscillations characterizing the strong coupling regime, but now the period is longer. As we will (a)

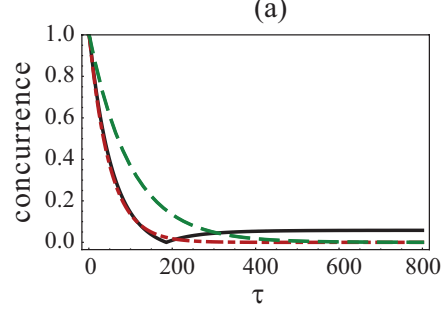

(b)

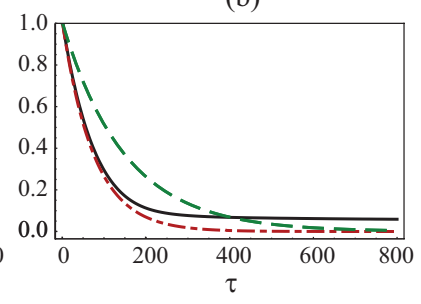

FIG. 1: (Color online) Time evolution of the concurrence in the bad cavity limit $(R=0.1)$ with $s=0$ and $\phi=0$, for the cases of i) maximal stationary value, when $r_{1}=\sqrt{3} / 2$ (black solid line), ii) symmetrical coupling $r_{1}=1 / \sqrt{2}$ (red dot-dashed line), and iii) only one coupled atom $r_{1}=0,1$ (green dashed line). For each of such cases, we describe the entanglement dynamics in two different coupling regime: the resonant limit (left plot) and for $\delta_{1}=\delta_{2}=0.7 \lambda$ (right plot).

see in Sec. III B, the generation of a high degree of entanglement in the dispersive regime for initially separable state can be achieved also in the good cavity limit. However it is remarkable that already in the bad cavity limit, values of concurrence close to one can be generated. Our approach generalizes the results obtained for the dispersive regime in Ref. [22] in the ideal cavity limit to the more realistic case of cavity losses.

\section{B. Good cavity limit - Entanglement quantum beats}

In the strong coupling case entanglement oscillations are present for any initial atomic state. Moreover, for 


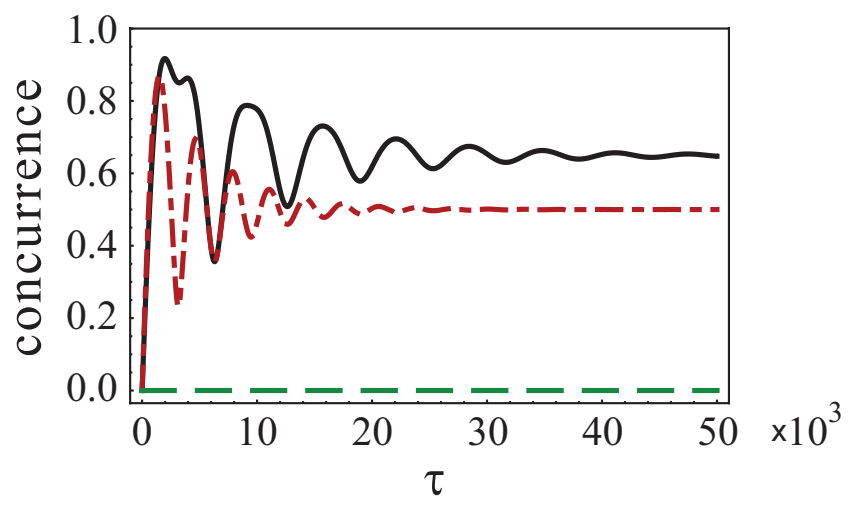

FIG. 2: (Color online) Time evolution of the concurrence in the bad cavity limit $(R=0.1)$ with $s=1$, for the cases of i) maximal stationary value, $r_{1}=\sqrt{3} / 2$ (black solid line), ii) symmetrical coupling $r_{1}=1 / \sqrt{2}$ (red dot-dashed line), and iii) only one coupled atom $r_{1}=0,1$ (green dashed line). All of the plots describe the dispersive regime with $\delta_{1}=\delta_{2}=10 \lambda$.

$\delta \approx \lambda \ll \mathcal{R}$, when both atoms are effectively coupled to the cavity field, i.e., $r_{1} \neq 0,1$, the dynamics of the concurrence is characterized by the occurrence of quantum beats, as shown in Fig. 3. For initially entangled states this phenomenon is more evident for $\phi=\pi$ because the value of stationary entanglement in this case is higher and the behavior of the concurrence is more regular.

In order to better understand the origin of these entanglement beats, we consider the case $s=1$ and $r_{1}=1 / \sqrt{2}$. For these values of the parameters, and for $\delta \approx \lambda \ll \mathcal{R}$, the expression of the concurrence can be written as follows,

$$
C(t) \approx \frac{1}{2} \sqrt{1+e^{-2 \lambda t} \cos (\mathcal{R} t)^{4}-2 e^{-\lambda t} \cos (\mathcal{R} t)^{2} \cos (\delta t)} .
$$

The term

$$
\cos (\mathcal{R} t)^{2} \cos (\delta t)=\frac{1}{2} \cos (\delta t)[1+\cos (2 \mathcal{R} t)]
$$

in Eq. (29), describing an oscillation at frequency $2 \mathcal{R}$ modulated by a slower one with frequency $\delta$, is responsible for the occurrence of the quantum beats.

To gain insight in the physical processes characterizing the dynamics, we consider the energy spectrum of the dressed states in the off-resonant case but in the absence of damping, as shown in Fig. 4. The diagonalization of the Tavis-Cummings Hamiltonian [See Eq. (39) in Appendix B] yields the dressed states

$$
\begin{aligned}
\left|\phi_{+}\right\rangle & =\frac{1}{\sqrt{\omega_{-}^{2}+\mathcal{R}^{2}}}\left(-\mathcal{R}\left|\psi_{+}\right\rangle|0\rangle_{R}+\omega_{-}|00\rangle|1\rangle_{R}\right), \\
\left|\phi_{-}\right\rangle & =\frac{1}{\sqrt{\omega_{+}^{2}+\mathcal{R}^{2}}}\left(-\mathcal{R}\left|\psi_{+}\right\rangle|0\rangle_{R}+\omega_{+}|00\rangle|1\rangle_{R}\right), \\
\left|\phi_{0}\right\rangle & =\left|\psi_{-}\right\rangle|0\rangle_{R} .
\end{aligned}
$$

The corresponding eigenenergies are given by

$$
\begin{aligned}
& \omega_{ \pm}=\frac{1}{2}\left(\delta \pm \sqrt{4 \mathcal{R}^{2}+\delta^{2}}\right) \\
& \omega_{0}=\delta
\end{aligned}
$$

where $\mathcal{R}=g \alpha_{T}$ is the vacuum Rabi frequency and $\delta$ is the qubits-cavity detuning.

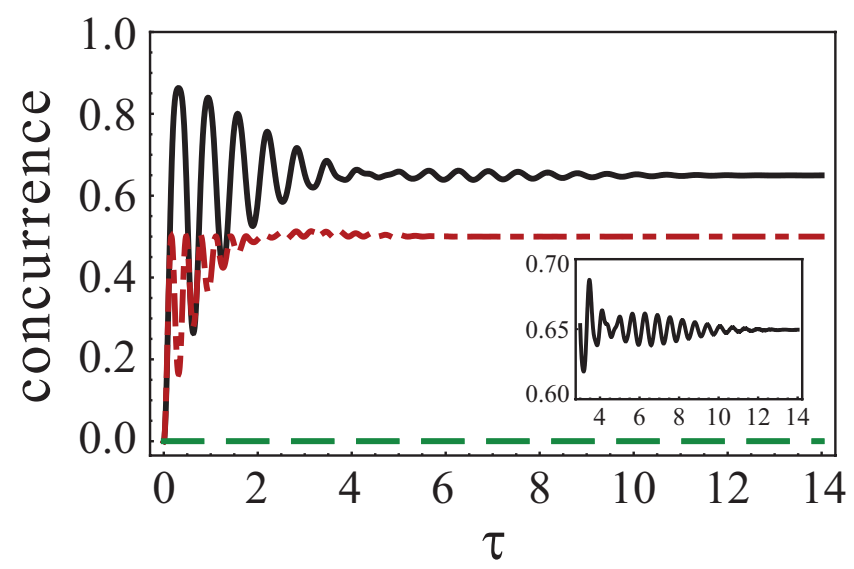

FIG. 3: (Color online) Time evolution of the concurrence in the good cavity limit $(R=10)$ with $s=1$, for the cases of i) maximal stationary value $r_{1}=\sqrt{3} / 2$ (black solid line), ii) symmetrical coupling $r_{1}=1 / \sqrt{2}$ (red dot-dashed line), and iii) only one coupled atom $r_{1}=0,1$ (green dashed line). The curves are drawn for small detuning, $\delta_{1}=\delta_{2}=0.7 \lambda$; thus, outside the dispersive region. The inset shows the entanglement beat for the case i).

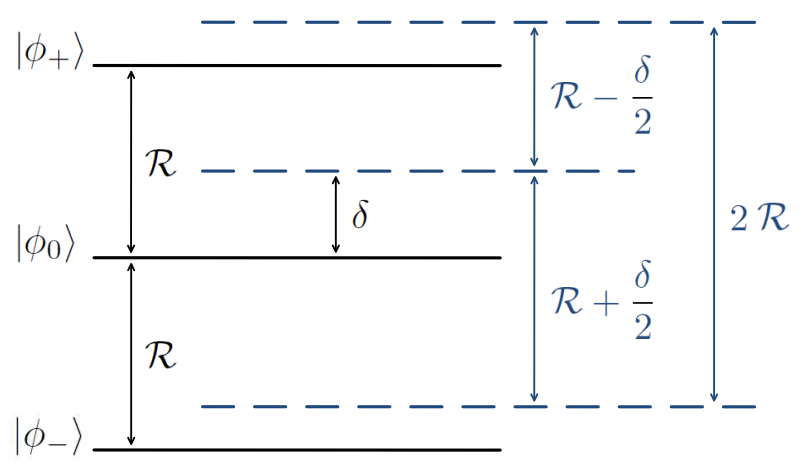

FIG. 4: (Color online) Energy spectrum of the dressed qubitphoton states in the case of small detuning (blue dashed line) and in the resonant coupling case (black solid line).

On the other hand, the unperturbed states can be expressed as a superposition of the $\left|\phi_{ \pm}\right\rangle,\left|\phi_{0}\right\rangle$ eigenstates, with probability amplitudes evolving at frequencies $\omega_{ \pm}$ and $\omega_{0}$. The effect of the detuning is a shift of the qubitscavity energy levels, thus the qubits-field coupling gives 
(a)
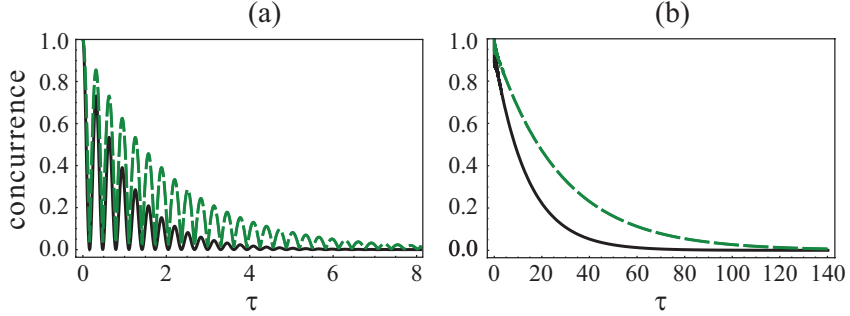

FIG. 5: (Color online) Time evolution of the concurrence in the good cavity limit $(R=10)$, with $s=0$ and $\phi=0$, for the cases of i) symmetrical coupling $r_{1}=1 / \sqrt{2}$ (black solid line), and ii) only one coupled atom $r_{1}=0,1$ (green dashed line). The two plots describe two different detuning regions: $\delta_{1}=\delta_{2}=0.7 \lambda$ (left) and $\delta_{1}=\delta_{2}=50 \lambda$ (right).

rise to a reversible energy exchange between unperturbed state at frequencies $2 \mathcal{R}, \mathcal{R}-\delta / 2$ and $\mathcal{R}+\delta / 2$. This is clearly seen, e.g., from the time evolution of the populations

$$
\begin{aligned}
\left|c_{2}(t)\right|^{2} & =\left|\left\langle 01\left|\left\langle 0\left|e^{-i H t}\right| 01\right\rangle\right| 0\right\rangle\right|^{2} \\
& =r_{1}^{4}+\frac{r_{2}^{4}}{2}[1+\cos (2 \mathcal{R} t)]+2 r_{1}^{2} r_{2}^{2} \cos (\mathcal{R} t) \cos \left(\frac{\delta}{2} t\right) .
\end{aligned}
$$

The equation above contains a term oscillating at frequency $2 \mathcal{R}$, coming from the coupling between the dressed states $\left|\phi_{+}\right\rangle$and $\left|\phi_{-}\right\rangle$, and a term oscillating at frequency $\mathcal{R}$ modulated by $\delta$ coming from the interference between the oscillations at frequencies $\mathcal{R}-\delta / 2$ and $\mathcal{R}+\delta / 2$ that couple the states $\left|\phi_{+}\right\rangle-\left|\phi_{0}\right\rangle$ and $\left|\phi_{-}\right\rangle-\left|\phi_{0}\right\rangle$, respectively.

In the discussion above we have disregarded the cavity losses. When they are taken into account one sees that the dressed energy splitting is resolved, and therefore the quantum beats will be visible, if $2 \mathcal{R}$ is larger than the decay width $\lambda$. This is achieved in strong coupling regime. Therefore, one does not observe quantum beats in the bad cavity case.

We conclude this section studying how the detuning influences the decay of entanglement, for an initially maximally entangled state of the system, and the reservoirinduced entanglement generation, for an initial factorized state.

When only one of the two qubits is effectively coupled to the cavity field, i.e. for $r_{1}=0,1$, for maximally entangled initial states $(s=0)$ in the resonant regime, $\delta=0$, the system performs damped oscillations between the states $\left|\psi_{+}\right\rangle$and $\left|\psi_{-}\right\rangle$, which are equally populated at the beginning. Hence entanglement revivals with maximum amplitude are present in the dynamics, as shown in Fig. 5 (a). Increasing the detuning, the amplitude of the oscillations decreases and the revivals disappear, while the frequency does not change appreciably, [See Fig. 5 (a)]. In this case the expression of the concurrence for small values of the detuning can be written as

$$
C(t)=|\mathcal{E}| \approx e^{-\lambda t / 2} \sqrt{\cos (\mathcal{R} t)^{2}+\frac{\delta^{2}+\lambda^{2}}{4 \mathcal{R}^{2}} \sin (\mathcal{R} t)^{2}-\frac{\lambda}{\mathcal{R}} \sin (\mathcal{R} t) \cos (\mathcal{R} t)}
$$

while for greater values of the detuning, the oscillations completely disappear and the concurrence decays exponentially

$$
C(t)=|\mathcal{E}| \approx e^{-\frac{\mathcal{R}^{2}}{\delta^{2}} \lambda t}
$$

as shown in Fig. 5 (b).

Finally, we note that, similarly to the behavior discussed in the bad cavity limit, when the qubits are initially in a factorized state, the presence of the detuning enhances the generation of entanglement at short times compared to the resonant coupling case, as illustrated in Fig. 2. In general, in the strongly dispersive regime, the qubits do not exchange energy with the cavity, which is only virtually excited. Thus a high degree of reservoirinduced entanglement can be generated both in the good and in the bad cavity limits.

\section{OFF-RESONANT ENTANGLEMENT IN THE NON-SUBRADIANT SCENARIO}

In this section, we analyze the more general situation in which the transition frequencies of the qubits are different, $\omega_{1} \neq \omega_{2}$, and both qubits are off-resonant with the cavity field. Due to the absence of a subradiant state, even a small value of the detunings $\delta_{1}, \delta_{2} \ll \mathcal{R}$ contributes to accelerate the decay of entanglement for every initial states. For an initially factorized state, in the bad cavity limit, the entanglement initially created via the interaction with the reservoir is rapidly destroyed as time evolves. In the good cavity limit entanglement oscillations are present and also quantum beats of entanglement can be observed for $\delta_{1}, \delta_{2} \approx \lambda \ll \mathcal{R}$.

We now consider in more detail the case in which the two qubits frequencies are symmetrically detuned from the central peak of the Lorentzian spectrum describing the field inside the cavity. In the dispersive region $\delta \gg \mathcal{R}$, and for initially entangled states $(s=0)$, the concurrence vanishes without manifesting a dominant depen- 
(a)
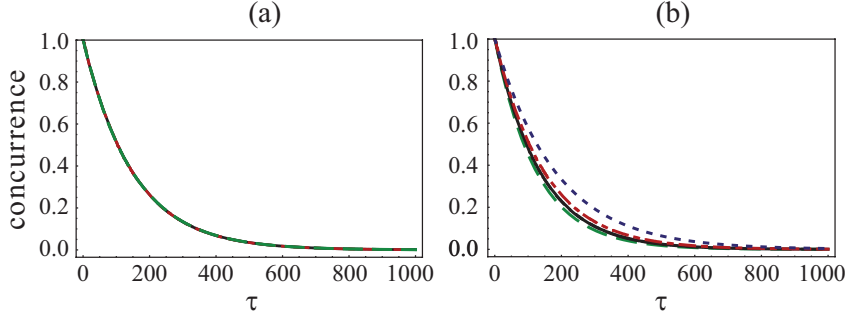

FIG. 6: (Color online) Time evolution of the concurrence in the bad cavity limit $(R=0.1)$, with $s=0$ and $\phi=0$, for the cases of i) maximal stationary value, corresponding to $r_{1}=\sqrt{3} / 2$ (black solid line), ii) symmetrical coupling $r_{1}=1 / \sqrt{2}$ (red dot-dashed line), and iii) only one coupled atom $r_{1}=1$ (green dashed line) and $r_{1}=0$ (blue dotted line). Two different detuning pairs are represented: the symmetrical detuning with $\delta_{1}=-0.7 \lambda, \delta_{2}=0.7 \lambda$ (left plot) and the asymmetrical detuning with $\delta_{1}=-0.5 \lambda, \delta_{2}=0.9 \lambda$ (right plot).

dence from $r_{1}$ and $\phi$. In other words, all the states initially entangled decay following the same behavior in such regime, as shown in Fig. 6 (a). This is in contrast to what we observed in all other regimes, where a dependence on the value of $r_{1}$ is present. We stress once more that this feature seems to occur only for the case of symmetric detuning. Indeed, when introducing a small asymmetry in the value of the detunings the behavior of the concurrence shows again a dependence on the parameter $r_{1}$, as illustrated in Fig. 6 (b).

In order to understand the peculiar behavior of the concurrence in the dispersive regime and for symmetric detunings we once more start by neglecting the effect of the cavity losses and use the dispersive Hamiltonian given by Eq. (24). For symmetrical detunings $\delta_{1}=-\delta_{2}$ this equation takes the form

$$
H_{e f f}=-\frac{\mathcal{R}^{2} r_{1}^{2}}{\delta} \sigma_{+}^{(1)} \sigma_{-}^{(1)}+\frac{\mathcal{R}^{2} r_{2}^{2}}{\delta} \sigma_{+}^{(2)} \sigma_{-}^{(2)},
$$

with $\delta=\left|\delta_{1}\right|=\left|\delta_{2}\right|$.

Comparing Eq. (37) with Eq. (24) we notice that the terms describing the effective dipole-dipole coupling induced by the cavity mode are here absent. Therefore the only remaining effect is the entanglement decay induced by the cavity losses. The decay rate, however, does not depend on the relative coupling parameter $r_{1}$ but only on the total coupling strength $\alpha_{T}$ via the vacuum Rabi frequency $\mathcal{R}$. This explains why, even when the cavity losses are taken into account, the time evolution of the concurrence for symmetric detunings does not depend on $r_{1}$. When a small asymmetry in the detunings is introduced, the dipole-dipole effective coupling terms are nonzero and, due to the presence of $r_{1}$ and $r_{2}$ in the effective dipole-dipole coupling strength, the dynamics becomes again dependent on $r_{1}$.

\section{SUMMARY AND CONCLUSIONS}

In this paper we have provided a complete analysis of the exact dynamics of the entanglement for two qubits interacting with a common zero-temperature reservoir in the off-resonant case. We have presented a general analytical solution for the two-qubit dynamics without performing the Born-Markov approximation. In the case of a Lorentzian spectrum, describing, e.g., the electromagnetic field inside a single mode lossy cavity, we have obtained explicit expressions for the reduced density matrix and for the concurrence. The availability of the exact solution allowed us to look at the entanglement dynamics both in the weak coupling (bad cavity) and in the strong coupling (good cavity) limits.

If the two qubits are initially disentangled, the interaction with the common reservoir generates entanglement. Our results demonstrate that a high degree of entanglement can be generated in this way, especially in the dispersive regime, and even in the bad cavity limit. For initially entangled states, the concurrence decay is slowed down when the qubits are detuned from the peak of the Lorentzian. In this case, indeed, the cavity losses affect less the atoms dynamics since the effective atom-atom interaction is mediated by virtual photon exchange.

In general, the entanglement dynamics is strongly sensitive to the relative coupling parameter $r_{1}$, indicating how strongly each of the two qubit is individually coupled to the e.m. field. Only when the qubits frequencies are symmetrically detuned from the main cavity frequency, in the dispersive regime, the dependence on the relative coupling disappears. Finally we have discovered that, in the strong coupling regime, for intermediate values of the detuning, the dynamics of the concurrence shows the occurrence of quantum beats. We have given a physical interpretation of this phenomenon in terms of the quantum interference between the transitions among the dressed states of the atomic system.

We believe that our results contribute in shedding light on the behavior of quantum entanglement in realistic conditions, that is when the effect of the environment on the quantum system is taken into account. For this reason they have both a fundamental and an applicative value and they indicate how rich the dynamics of this system can be. The model we have studied can be employed to describe both trapped ions in optical cavities 25] and circuit cavity QED dynamics [26, 27, 28]. In both physical contexts, the observation of the effects we have discussed should be achievable with the current experimental technologies.

\section{ACKNOWLEDGEMENTS}

S.M., K.-A.S., and J.P. acknowledge financial support from the Academy of Finland Projects No. 108699, No. 115682 and No. 115982, the Magnus Ehrnrooth Foundation, and the Väisälä Foundation. F.F. acknowledges 
financial support from the CIMO Project No. TM-085425 and thanks N. Lo Gullo and R. L. Zaffino for useful discussions, and S.M. and all of the Quantum Optics Group members at the University of Turku for the kind hospitality. Finally, the authors acknowledge W. Lange and B. Garraway for discussions on the experimental implementation with ion-cavity QED set up.

\section{Appendix A - Analytic solution for the probability amplitudes}

In this Appendix we briefly discuss the structure of the analytical solutions of Eqs. (13)-(14) for the probability amplitudes $c_{1,2}(t)$ and how they can be obtained applying the Laplace transform method. We note that the solutions obtained in this way are exact since we do not perform any kind of approximation.

The solution of the Laplace transformed amplitudes $\widetilde{c}_{1,2}(s)$, obtained from Eqs. (15)-(16) can be written as the sum of three ratios having denominators $\left(s-s_{i}\right)$, where $s_{i}$ are the roots of the cubic equation.

$$
s^{3}+A_{j} s^{2}+B_{j} s+C_{j}=0, \quad(j=1,2)
$$

where

$$
\begin{aligned}
& A_{1,2}=\lambda+i\left(\delta_{2,1}-2 \delta_{1,2}\right) \\
& B_{1,2}=\mathcal{R}^{2}-\delta_{1,2}^{2}+\delta_{1} \delta_{2}+i\left(\delta_{2,1}-\delta_{1,2}\right) \lambda, \\
& C_{1,2}=i \mathcal{R}^{2} r_{1,2}^{2}\left(\delta_{2,1}-\delta_{1,2}\right) .
\end{aligned}
$$

The amplitudes $c_{1,2}(t)$, obtained by inverse Laplace transform will then be the sum of three damped oscillating terms having, in general, a complicated structure. Only for the case $\omega_{1}=\omega_{2}$ a simple analytical expressions for the probability amplitudes can be obtained, whereas in the general case there is no simple solution. This is because, when $\omega_{1}=\omega_{2}$ the cubic equation can be written as a product of polynomials of first and second order having always one root coincident with zero. In this case one can write the amplitudes in the simple form given by Eqs. (18)-(19).

\section{Appendix B - Effective dispersive Hamiltonian}

The Hamiltonian describing the interaction between two-qubit systems and the quantized cavity mode is given by

$$
\begin{aligned}
H= & \sum_{j=1}^{2} \omega_{j} \sigma_{+}^{(j)} \sigma_{-}^{(j)}+\omega_{c} b^{\dagger} b \\
& +\left[g\left(\alpha_{1} \sigma_{+}^{(1)}+\alpha_{2} \sigma_{+}^{(2)}\right) b+\text { h.c. }\right] .
\end{aligned}
$$

To obtain the effective Hamiltonian describing the interaction with the cavity in the dispersive regime, one can apply the canonical transformation defined by the unitary operator [23, 24]

$$
e^{\alpha S}=e^{-\sum_{j=1}^{2} \frac{\mathcal{R} r_{j}}{\delta_{j}}\left(b \sigma_{+}^{(j)}-b^{\dagger} \sigma_{-}^{(j)}\right)}
$$

with $\mathcal{R} r_{j}=g \alpha_{j}$. This procedure is correct to the second order in the coupling to the cavity, and, limiting ourselves to this approximation, we can write the effective Hamiltonian as follows

$$
H_{e f f}=e^{\alpha S} H e^{-\alpha S} \simeq H+\alpha[S, H]+\frac{\alpha^{2}}{2}[S,[S, H]] .
$$

Assuming that the cavity field is initially in the vacuum state, $H_{\text {eff }}$ takes the form

$H_{e f f}=\sum_{j=1}^{2} \frac{\mathcal{R}^{2} r_{j}^{2}}{\delta_{j}} \sigma_{+}^{(j)} \sigma_{-}^{(j)}+\frac{\mathcal{R}^{2} r_{1} r_{2}}{2 \delta_{j}}\left(\sigma_{+}^{(1)} \sigma_{-}^{(2)}+\sigma_{+}^{(2)} \sigma_{-}^{(1)}\right)$

where the terms proportional to $\sigma_{+}^{(j)} \sigma_{-}^{(j)}$ describe the Stark shifts due to the dispersive interaction, while the last two terms describe the dipole-dipole coupling between the two atoms induced by the cavity mode through the exchange of virtual cavity photons.

\section{Appendix C - Approximate expressions of the concurrence}

In this Appendix we derive approximate expressions for the amplitudes $c_{1,2}(t)$ in the case of large (and equal) detuning $\delta \gg \lambda \gg \mathcal{R}$.

For this purpose, we expand the term $\Omega=$ $\sqrt{\lambda^{2}-\Omega_{R}^{2}-i 2 \delta \lambda}$ as follows,

$$
\Omega \approx \lambda\left(1-\frac{2 \mathcal{R}^{2}}{\delta^{2}}\right)-i\left(\delta+\frac{2 \mathcal{R}^{2}}{\delta}\right) .
$$

The temporal evolution described by $\mathcal{E}(t)$ can then be written as

$$
\begin{aligned}
\mathcal{E}(t) & \approx e^{-(\lambda-i \delta) t / 2}\left[\cosh \left(\frac{\Omega t}{2}\right)+\sinh \left(\frac{\Omega t}{2}\right)\right] \\
& \approx e^{-\frac{\mathcal{R}^{2}}{\delta^{2}}(\lambda+i \delta) t}
\end{aligned}
$$

For the sake of simplicity we consider here the case $s=1$ and $r_{1}=1 / \sqrt{2}$. However the time evolution of the concurrence has features in common with all of the other cases:

$$
\begin{aligned}
C(t) & =2\left|c_{1}(t)\right|\left|c_{2}(t)\right| \\
& =\frac{1}{2} \sqrt{\left(1+|\mathcal{E}(t)|^{2}\right)^{2}-(2 \operatorname{Re}[\mathcal{E}(t)])^{2}} \\
& \approx \frac{1}{2} \sqrt{1+e^{-4 \frac{\mathcal{R}^{2}}{\delta^{2}} \lambda t}-2 e^{-2 \frac{\mathcal{R}^{2}}{\delta^{2}} \lambda t} \cos \left(2 \frac{\mathcal{R}^{2}}{\delta} t\right)} .
\end{aligned}
$$


On other hand, for small detunings of the order of $\lambda$, outside the dispersive region $\delta \ll \mathcal{R}$, the approximate form of $\Omega$ is given by

$$
\Omega \approx \frac{\lambda \delta}{2 \mathcal{R}}-i 2 \mathcal{R}
$$

so that the time evolution is described by the function

$$
\begin{aligned}
\mathcal{E}(t) & \approx e^{-(\lambda-i \delta) t / 2}\left[\cosh \left(\frac{\Omega t}{2}\right)-\frac{\delta+i \lambda}{2 \mathcal{R}} \sinh \left(\frac{\Omega t}{2}\right)\right] \\
& \approx e^{-(\lambda-i \delta) t / 2}\left[\cos (\mathcal{R} t)-\frac{\lambda}{2 \mathcal{R}} \sin (\mathcal{R} t)+i \frac{\delta}{2 \mathcal{R}} \sin (\mathcal{R} t)\right]
\end{aligned}
$$

[1] T. Yu and J. H. Eberly, Phys. Rev. Lett. 93, 140404 (2004); T. Yu and J. H. Eberly, Phys. Rev. Lett. 97, 140403 (2006); J. H. Eberly and T. Yu, Science 316, 555 (2007).

[2] M. P. Almeida et al., Science 316, 579 (2007).

[3] J. Laurat, K. S. Choi, H. Deng, C. W. Chou, and H. J. Kimble, Phys. Rev. Lett. 99, 180504 (2007).

[4] P. Marek, J. Lee, and M. S. Kim, Phys. Rev. A 77, 032302 (2008).

[5] A. Al-Qasimi and D. F. V. James, Phys. Rev. A 77, 012117 (2008).

[6] B. Bellomo, R. Lo Franco, and G. Compagno, Phys. Rev. Lett. 99, 160502 (2007); B. Bellomo, R. Lo Franco, and G. Compagno, Phys. Rev. A 77, 032342 (2008).

[7] I. Sainz and G. Björk, Phys. Rev. A 76, 042313 (2007).

[8] C. E. López, G. Romero, F. Lastra, E. Solano, and J. C. Retamal, Phys. Rev. Lett. 101, 080503 (2008).

[9] F. Benatti, R. Floreanini, and M. Piani, Phys. Rev. Lett. 91, 070402 (2003).

[10] F. Benatti, and R. Floreanini, Int. J. Quant. Inf. 4(3), 395 (2006).

[11] S. Oh and J. Kim, Phys. Rev. A 73, 062306 (2006).

[12] R.-F. Liu and C.-C. Chen, Phys. Rev. A 74, 024102 (2006).

[13] J.-H. An, S.-J. Wang, and H.-G. Luo, Physica A 382, 753 (2007).
Therefore, for the case $s=1$ and $r_{1}=1 / \sqrt{2}$ the time evolution of the concurrence is given by

$$
\begin{aligned}
C(t) & =\frac{1}{2} \sqrt{\left(1+|\mathcal{E}(t)|^{2}\right)^{2}-(2 \operatorname{Re}[\mathcal{E}(t)])^{2}} \\
& \approx \frac{1}{2} \sqrt{1+e^{-2 \lambda t} \cos (\mathcal{R} t)^{4}-2 e^{-\lambda t} \cos (\mathcal{R} t)^{2} \cos (\delta t)}
\end{aligned}
$$

[14] S. Natali and Z. Ficek, Phys. Rev. A 75, 042307 (2007).

[15] J. Dajka, and J. Łuczka, Phys. Rev. A 77, 062303 (2008).

[16] Z. Ficek and R. Tanaś, Phys. Rev. A 74, 024304 (2006); Z. Ficek and R. Tanaś, Phys. Rev. A 77, 054301 (2008).

[17] C. Anastopoulos, S. Shresta, and B. L. Hu, arXiv:quant-ph/0610007 2 .

[18] S. Maniscalco, F. Francica, R. L. Zaffino, N. Lo Gullo, and F. Plastina, Phys. Rev. Lett. 100, 090503 (2008).

[19] G. M. Palma, K.-A. Suominen, A. K. Ekert, Proc. Roy. Soc. Lond. A 452, 567 (1996).

[20] P. Zanardi, and M. Rasetti, Phys. Rev. Lett. 79, 3306 (1997); P. Zanardi, Phys. Rev. A 56, 4445 (1997).

[21] W. K. Wootters, Phys. Rev. Lett. 80, 2245 (1998).

[22] S. B. Zheng, G. C. Guo, Phys. Rev. Lett. 85, 2392 (2000).

[23] A. Blais, R.-S. Huang, A. Wallraff, S. M. Girvin, and R. J. Schoelkopf, Phys. Rev. A 69, 062320 (2004).

[24] L. Roa, R. Pozo-González, M. Schaefer, and P. UtrerasSM, Phys. Rev. A 75, 062316 (2007).

[25] G. R. Guthöhrlein et al., Nature 41, 449 (2001); P. Maunz et al., Nature 50, 428 (2004).

[26] A. Wallraff et al., Nature 431, 162 (2004).

[27] M.A. Sillanpää, J. I. Park, and R. W. Simmonds, Nature 449, 438 (2007).

[28] J. Majer et al., Nature 449, 443 (2007). 